\title{
The Concepts of Well-Posedness and Stability in Different Function Spaces for the 1D Linearized Euler Equations
}

\author{
Stefan Balint ${ }^{1}$ and Agneta M. Balint ${ }^{2}$ \\ ${ }^{1}$ Department of Computer Science, West University of Timisoara, Boulevard Vasile Pârvan 4, 300223 Timisoara, Romania \\ ${ }^{2}$ Department of Physics, West University of Timisoara, Boulevard Vasile Pârvan 4, 300223 Timisoara, Romania \\ Correspondence should be addressed to Agneta M. Balint; balint@physics.uvt.ro
}

Received 30 August 2013; Revised 8 December 2013; Accepted 15 December 2013; Published 12 January 2014

Academic Editor: Abdullah Alotaibi

Copyright (c) 2014 S. Balint and A. M. Balint. This is an open access article distributed under the Creative Commons Attribution License, which permits unrestricted use, distribution, and reproduction in any medium, provided the original work is properly cited.

\begin{abstract}
This paper considers the stability of constant solutions to the $1 \mathrm{D}$ Euler equation. The idea is to investigate the effect of different function spaces on the well-posedness and stability of the null solution of the 1D linearized Euler equations. It is shown that the mathematical tools and results depend on the meaning of the concepts "perturbation," "small perturbation," "solution of the propagation problem," and "small solution, that is, solution close to zero," which are specific for each function space.
\end{abstract}

\section{Motivation of the Mathematical Considerations}

Due to the practical importance of the sound attenuation in case of the turbofan aircraft engines, in the last years more than six hundred papers, reporting experimental and theoretical results on the subject, were published. The papers refereed here and those refereed herein concern acoustic perturbation propagation in a gas flowing through a lined duct and represent just a very small part of the literature concerning the subject.

For describing an instantaneous acoustic perturbation propagation, the authors consider the solution of the nonlinear Euler equations (without source) governing the gas flow. After that, the nonlinear Euler equations are linearized at the specified solution and the homogeneous linearized Euler equations are derived. It is commonly accepted that those equations govern the propagation of an instantaneous acoustic perturbation (called frequently also initial value perturbations). More precisely, it is assumed that if at the moment, let us say $t=0$, an instantaneous acoustic perturbation occurs, then its propagation is described by that solution of the homogeneous linearized Euler equations which satisfies on the duct wall the boundary condition and at $t=0$ it is equal to the perturbation in discussion.

For describing a source-produced permanent time harmonic perturbation propagation, the mathematical objects describing the perturbation are added as right-hand members to the homogeneous linearized Euler equations. It is commonly accepted that the so-obtained nonhomogeneous linearized Euler equations govern the propagation of the source-produced permanent time harmonic perturbation in discussion. More precisely, it is assumed that if starting at the moment, let us say $t=0$, a source begins to produce permanent time harmonic perturbation, then the propagation of this perturbation is described by that solution of the nonhomogeneous linearized Euler equations which is equal to zero for $t \leq 0$ and verifies on the duct wall the boundary conditions.

It turns that in [1] for a large class of impedance lining models (i.e., boundary conditions) the above presented initial-boundary value problem was declared "illposed" because the set of the exponential growth rates of the solutions of the homogeneous linearized Euler equations, satisfying the considered boundary conditions, is unbounded from above. Later in [2-4] considerable efforts were made 
for modifying the boundary conditions in order to make the problem "well posed" in the sense defined in [1]. Here it has to be mentioned that in [1] a concept of "well posed differential equation" is defined. This is different from the concept of "well-posed problem" usually in mathematics $[5,6]$ and introduced by Hadamard long time ago.

Definition 1. Following Hadamard, for a given class of instantaneous initial value perturbations (permanent source-produced time harmonic perturbations, resp.) one calls the perturbation propagation problem well posed if there is a unique solution to the problem and the solution varies continuously with the initial data (source amplitude, resp.).

In [7] it was shown that the concept introduced in [1] is confusing, because the equation considered in [1], depending on the function space, can be ill-posed or can be well posed. In fact the concept introduced in [1] is the transcription of the behavior of an example of a semigroup of contractions of class $C_{0}$ which act in a precise function space (see $[1,8]$ pages 240-241 referred in [1]). In this situation, according to [8], the resolvent operator of the infinitesimal generator exists in a half plane of the form $\operatorname{Re} z>\mu$ and the Laplace transform can be considered for every solution of the homogeneous equation.

The objective of the present work is to underline that the precise description of the function space is crucial even in the case of the $1 \mathrm{D}$ gas flow model, where the wall and the lining effect are absent. For the achievement of this objective the linear stability analysis of the constant 1D gas flow with respect to the initial value and source-produced permanent time harmonic perturbations is presented in four different function spaces, revealing significant differences. For instance, in the topological function space $X_{4}$ the propagation problem of the initial value perturbation is well posed, but that of the source produced perturbation is ill posed; in the topological function space $X_{1}$ in which the origin has not absorbing neighborhoods, stability and strictly positive growth rate coexists in contrast to the case of topological vector spaces $X_{2}, X_{3}$.

\section{The 1D Gas Flow Model}

In the $1 \mathrm{D}$ gas flow model the nonlinear Euler equations governing the flow of an inviscid, compressible, nonheat conducting, isentropic, perfect gas, according to [9], are

$$
\begin{aligned}
& \frac{\partial u}{\partial t}+u \cdot \frac{\partial u}{\partial x}+\frac{1}{\rho} \cdot \frac{\partial p}{\partial x}=0 \\
& \frac{\partial \rho}{\partial t}+u \cdot \frac{\partial \rho}{\partial x}+\rho \cdot \frac{\partial u}{\partial x}=0
\end{aligned}
$$

Here, $t$ is time, $u$ is velocity along the $O x$ axis, $p$ is pressure, and $\rho$ is density. Equation (1) are considered for $x \in R^{\prime}$ and $t \geq 0$. It is assumed that $p, \rho$ and the absolute temperature $T^{\prime}$ satisfy the equation of state of the perfect gas:

$$
p=\rho \cdot R \cdot T^{\prime}
$$

with $R=c_{p}-c_{v} ; c_{p}, c_{v}$ being the specific heat capacities at constant pressure and constant volume, respectively. Let $u \equiv U_{0}=$ const $>0, \rho \equiv \rho_{0}=$ const $>0, p \equiv p_{0}=$ const $>0$ be a constant solution of the system of partial differential equations (SPDE) (1). According to (2), $p_{0}=\rho_{0} \cdot R \cdot T_{0}^{\prime}$ and the associated isentropic sound speed $c_{0}$ verifies $c_{0}^{2}=\gamma \cdot\left(p_{0} / \rho_{0}\right)=$ $\gamma \cdot R \cdot T_{0}^{\prime}$, where $\gamma=c_{p} / c_{v}$.

Linearizing (1) at $u=U_{0}, \rho=\rho_{0}, p=p_{0}$ and using the perturbations $p^{\prime}, \rho^{\prime}$ of $p_{0}, \rho_{0}$ satisfying

$$
\left(\frac{\partial}{\partial t}+U_{0} \cdot \frac{\partial}{\partial x}\right)\left(p^{\prime}-c_{0}^{2} \rho^{\prime}\right)=0,
$$

the following system of homogeneous linear partial differential equations, for the perturbations $u^{\prime}, p^{\prime}$ of $U_{0}, p_{0}$, is obtained:

$$
\begin{gathered}
\frac{\partial u^{\prime}}{\partial t}+U_{0} \cdot \frac{\partial u^{\prime}}{\partial x}+\frac{1}{\rho_{0}} \cdot \frac{\partial p^{\prime}}{\partial x}=0, \\
\frac{\partial p^{\prime}}{\partial t}+U_{0} \cdot \frac{\partial p^{\prime}}{\partial x}+\gamma \cdot p_{0} \cdot \frac{\partial u^{\prime}}{\partial x}=0 .
\end{gathered}
$$

It is assumed that if at $t=0$ an acoustic perturbation occurs, then its propagation is given by that solution of (4) which at $t=0$ is equal to the perturbation in discussion. It is assumed also that if at $t=0$ a source begins to produce permanent time harmonic acoustic perturbation, then the propagation of this perturbation is given by that solution of the system of non-homogeneous linearized Euler equations:

$$
\begin{gathered}
\frac{\partial u^{\prime}}{\partial t}+U_{0} \cdot \frac{\partial u^{\prime}}{\partial x}+\frac{1}{\rho_{0}} \cdot \frac{\partial p^{\prime}}{\partial x}=Q_{1} \\
\frac{\partial p^{\prime}}{\partial t}+U_{0} \cdot \frac{\partial p^{\prime}}{\partial x}+\gamma \cdot p_{0} \cdot \frac{\partial u^{\prime}}{\partial x}=Q_{2}
\end{gathered}
$$

which is equal to zero for $t \leq 0$.

Here $Q_{1}, Q_{2}$ (functions or distributions) describe the source-produced permanent time harmonic acoustic perturbations, being equal to zero for $t \leq 0$ and periodic in $t$ for $t>0$.

Definition 2. The constant solution $u=U_{0}, p=p_{0}, \rho=\rho_{0}$ of (1) is linearly stable with respect to the initial value perturbation (to the source-produced permanent time harmonic perturbation, resp.) if the "solution $u^{\prime}(x, t), p^{\prime}(x, t)$ of $(4)$ " (of (5), resp.) "is small $=$ close to zero" all time $t \geq 0$ provided " $u^{\prime}(x, 0), p^{\prime}(x, 0)$ " $\left(\left(Q_{1}, Q_{2}\right)\right.$, resp. $)$ "is small = close to zero."

In other words, the constant solution of (1) is linearly stable if and only if the null solution of (4) is stable.

These stability concepts are not necessarily equivalent to the hydrodynamic stability defined in [10].

The precise meaning of the concepts: "perturbation," "small perturbation," "solution of the propagation problem," and "small solution" has to be designed by other definitions and there is some freedom here.

Using this freedom, in the following we present four different function spaces in order to reveal that in each of them the meaning of "perturbation," "small perturbation," "solution to the propagation problem," and "small solution" is specific and the results and mathematical tools are specific as well. 


\section{The First Function Space}

The set $X_{1}$ of the perturbations of the initial value (instantaneous perturbations) is the topological function space [11] of the couples $H=(F, G)$ of continuously differentiable functions $F, G: R^{\prime} \rightarrow R^{\prime}$ with respect to the usual algebraic operations and topology generated by the uniform convergence on $R^{\prime}$ [12].

A neighborhood of the origin $O$ is a set $V_{0}$ of couples from $X_{1}$ having the property that there exists $\varepsilon>0$ such that if for $H=(F, G) \in X_{1}$ we have $|F(x)|<\varepsilon$ and $|G(x)|<\varepsilon$ for any $x \in R^{\prime}$, then $H=(F, G) \in V_{0}$.

The set $V_{0}^{\varepsilon}$ defined by

$$
V_{0}^{\varepsilon}=\left\{H=(F, G) \in X_{1}:|F(x)|<\varepsilon,|G(x)|<\varepsilon \forall x \in R^{\prime}\right\}
$$

is a neighborhood of the origin.

The meaning of the concept "the perturbation $H=(F, G)$ $\in X_{1}$ is small" is that there exists $\varepsilon$ small such that $H \in V_{0}^{\varepsilon}$.

For a perturbation $H=(F, G) \in X_{1}$ (i.e., $u^{\prime}(x, o)=$ $\left.F(x), p^{\prime}(x, o)=G(x)\right)$ the couple of functions $H^{\prime}(x, t)=$ $\left(u^{\prime}(x, t), p^{\prime}(x, t)\right)$ given by

$$
\begin{aligned}
u^{\prime}(x, t)= & \frac{F\left[x-\left(U_{0}-c_{0}\right) t\right]+F\left[x-\left(U_{0}+c_{0}\right) t\right]}{2} \\
& +\frac{G\left[x-\left(U_{0}+c_{0}\right) t\right]-G\left[x-\left(U_{0}-c_{0}\right) t\right]}{2 c_{0} \rho_{0}}, \\
p^{\prime}(x, t)= & c_{0} \rho_{0} \cdot \frac{F\left[x-\left(U_{0}+c_{0}\right) t\right]-F\left[x-\left(U_{0}-c_{0}\right) t\right]}{2} \\
& +\frac{G\left[x-\left(U_{0}+c_{0}\right) t\right]+G\left[x-\left(U_{0}-c_{0}\right) t\right]}{2}
\end{aligned}
$$

is continuously differentiable and verifies (4) and the initial condition:

$$
\left(u^{\prime}(x, 0), p^{\prime}(x, 0)\right)=H^{\prime}(x, 0)=H(x)=(F(x), G(x)) .
$$

Proposition 3. In the class of the continuously differentiable functions, the couple of functions $\left(u^{\prime}(x, t), p^{\prime}(x, t)\right)$, given by (7), is the unique point wise (classical) solution of the initial value problem (4) and (8).

Proof. If $\left(u^{\prime}(x, t), p^{\prime}(x, t)\right)$ is a point wise (classical) solution of the initial value problem $(4),(8)$, then $v^{\prime}=u^{\prime}-\left(1 / c_{0} \rho_{0}\right) \cdot p^{\prime}$ and $q^{\prime}=u^{\prime}+\left(1 / c_{0} \rho_{0}\right) \cdot p^{\prime}$ satisfy the equalities

$$
\begin{gathered}
\left(\partial v^{\prime} / \partial t\right)+\left(U_{0}-c_{0}\right) \cdot\left(\partial v^{\prime} / \partial x\right)=0, \\
v^{\prime}(x, 0)=F(x)-\left(1 / c_{0} \rho_{0}\right) \cdot G(x), \\
\left(\partial q^{\prime} / \partial t\right)+\left(U_{0}+c_{0}\right) \cdot\left(\partial q^{\prime} / \partial x\right)=0, \\
q^{\prime}(x, 0)=F(x)+\left(1 / c_{0} \rho_{0}\right) \cdot G(x) .
\end{gathered}
$$

Hence, by using the method of characteristics, we obtain

$$
\begin{aligned}
& v^{\prime}(x, t)=F\left(x-\left(U_{0}-c_{0}\right) t\right)-\frac{1}{c_{0} \rho_{0}} \cdot G\left(x-\left(U_{0}-c_{0}\right) t\right) \\
& q^{\prime}(x, t)=F\left(x-\left(U_{0}+c_{0}\right) t\right)+\frac{1}{c_{0} \rho_{0}} \cdot G\left(x-\left(U_{0}+c_{0}\right) t\right) .
\end{aligned}
$$

Taking into account the equalities $u^{\prime}=(1 / 2)\left(v^{\prime}+q^{\prime}\right), p^{\prime}=$ $\left(c_{0} \rho_{0} / 2\right)\left(q^{\prime}-v^{\prime}\right)$, we deduce that $u^{\prime}(x, t), p^{\prime}(x, t)$ are given by the formula (7). So, it was shown that if $\left(u^{\prime}(x, t), p^{\prime}(x, t)\right)$ is a point wise solution of (4) and (8), then necessarily $u^{\prime}(x, t), p^{\prime}(x, t)$ are given by (7). The fact that the couple of functions given by (7) is a solution of (4), (8) is obtained by verification.

In other words, when the set of the perturbations of the initial value is $X_{1}$, then the initial value problem (4) and (8) has a unique classical (point wise) solution [9] given by (7).

If a sequence of perturbations $H_{n}=\left(F_{n}, G_{n}\right) \in X_{1}$ converges in $X_{1}$ to $H=(F, G) \in X_{1}$, then for any $t \geq 0$ (fixed) the sequence of the corresponding solutions $H_{n}^{\prime}(\cdot, t)$ belongs to $X_{1}$ and converges in $X_{1}$ to the solution $H^{\prime}(\cdot, t)$, corresponding to $H$.

This means that when the set of the initial value perturbations is the function space $X_{1}$, then the initial value problem (4) and (8) is well posed in sense of Hadamard $[5,6]$ on $[0, T]$, for any $T>0$.

In the following the couple of functions defined by (7) is considered to be the solution to the problem of propagation of the initial value perturbation for data in $X_{1}$.

The linear stability of the constant flow $U_{0}, p_{0}, \rho_{0}$ would mean that for any $\varepsilon>0$ there exists $\delta=\delta(\varepsilon)$ such that for any $H=(F, G) \in V_{0}^{\delta(\varepsilon)}$ the corresponding solution $H^{\prime}(x, t)$ (given by (7)) satisfies $H^{\prime}(\cdot, t) \in V_{0}^{\varepsilon}$ for any $t \geq 0$.

Concerning the linear stability the following statement holds.

Proposition 4. For any $\varepsilon>0$ and $\delta(\varepsilon)=\varepsilon / 2 \max \left(1+c_{0} \rho_{0}, 1+\right.$ $\left.\left(1 / c_{0} \rho_{0}\right)\right)$ if $H \in V_{0}^{\delta(\varepsilon)}$, then $H^{\prime}(\cdot, t) \in V_{0}^{\varepsilon}$ for $t \geq 0$.

Proof. If $H=(F, G) \in V_{0}^{\delta(\varepsilon)}$, then $|F(x)|<\delta(\varepsilon)$ and $|G(x)|<$ $\delta(\varepsilon)$ for any $x \in R^{\prime}$. Since $\delta(\varepsilon) \leq \varepsilon / 2\left(1+c_{0} \rho_{0}\right)$ and $\delta(\varepsilon) \leq$ $\varepsilon / 2\left(1+\left(1 / c_{0} \rho_{0}\right)\right)$ it follows that the following inequalities hold: $|F(x)|<\varepsilon / 2\left(1+c_{0} \rho_{0}\right),|F(x)|<\varepsilon / 2\left(1+\left(1 / c_{0} \rho_{0}\right)\right),|G(x)|<$ $\varepsilon / 2\left(1+c_{0} \rho_{0}\right)$ and $|G(x)| \leq \varepsilon / 2\left(1+\left(1 / c_{0} \rho_{0}\right)\right)$ for any $x \in R^{\prime}$.

By using (7) we obtain that for any $x \in R^{\prime}$ and $t \geq 0$ the following inequalities hold:

$$
\begin{aligned}
\left|u^{\prime}(x, t)\right|< & \frac{1}{2} \cdot 2 \cdot \frac{\varepsilon}{2\left(1+c_{0} \rho_{0}\right)}+\frac{1}{2 c_{0} \rho_{0}} \\
& \cdot 2 \cdot \frac{\varepsilon}{2\left(1+\left(1 / c_{0} \rho_{0}\right)\right)}=\varepsilon
\end{aligned}
$$




$$
\begin{aligned}
\left|p^{\prime}(x, t)\right|< & \frac{1}{2} \cdot 2 c_{0} \rho_{0} \cdot \frac{\varepsilon}{2\left(1+\left(1 / c_{0} \rho_{0}\right)\right)} \\
& +\frac{1}{2} \cdot \frac{2 \varepsilon}{2\left(1+c_{0} \rho_{0}\right)}=\varepsilon
\end{aligned}
$$

Hence, $H^{\prime}(\cdot, t) \in V_{0}^{\varepsilon}$ for any $t \geq 0$.

This means that the null solution of (4) is stable; that is, the constant solution $u=U_{0}, p=p_{0}, \rho=\rho_{0}$ of (1) is linearly stable with respect to the initial value perturbation with data from $X_{1}$.

On the other hand, for initial data from $X_{1}$ the set of the exponential growth rates of the solutions of the initial value problem (4) and (8) is the whole real axis and there exist also solutions whose exponential growth rate is equal to $+\infty$ (for instance, that of the solution corresponding to the initial data $\left.F(x)=G(x)=\exp \left(x^{2}\right)\right)$.

Here, the exponential growth rate of $H^{\prime}(x, t)=\left(u^{\prime}(x, t)\right.$, $\left.p^{\prime}(x, t)\right)$ is defined as

$$
\max \left\{\sup _{x} \varlimsup_{t \rightarrow \infty} \frac{\ln \left|u^{\prime}(x, t)\right|}{t}, \sup _{x} \varlimsup_{t \rightarrow \infty} \frac{\ln \left|p^{\prime}(x, t)\right|}{t}\right\} .
$$

The stability of the null solution of (4) (i.e., linear stability of the constant solution) in the presence of solutions having strictly positive or $+\infty$ growth rate can be surprising. That is because usually the stability of the null solution of the infinite dimensional linear evolutionary equations is analyzed in a function space in which the Hille-Yosida theory can be applied $[5,6,8,13]$, that is, Banach space or locally convex and sequentially complete topological vector spaces. In the topological function space $X_{1}$ the origin possesses neighborhoods which are not absorbing. This is the main reason while stability and strictly positive growth rate coexist.

For initial value perturbations from $X_{1}$, the very popular Briggs-Bers stability analysis $[14,15]$, in case of the problem (4), (8), cannot be applied. That is because in this context dispersion relations cannot be derived (for an arbitrary solution, the Fourier transform with respect to $x$ and the Laplace transform with respect to $t$ in this context do not exist). So, it is impossible to analyze the imaginary parts of the zeros of the dispersion relations as requires the Briggs-Bers stability criterion.

We consider now source-produced permanent time harmonic perturbations whose amplitudes belong to $X_{1}$. More precisely, perturbations for which the right-hand members $Q_{1}, Q_{2}$, appearing in (5), are of the form

$$
\begin{aligned}
& Q_{1}(x, t)=F(x) \cdot h(t) \cdot \sin \omega_{f} t \\
& Q_{2}(x, t)=G(x) \cdot h(t) \cdot \sin \omega_{f} t
\end{aligned}
$$

Here, $(F, G) \in X_{1}$ and represent the amplitude of the perturbation, $h(t)$ is Heaviside function, and $\omega_{f}>0$ is the angular frequency. Such a perturbation will be denoted by $P\left(F, G, \omega_{f}\right)$.
For the perturbation $P\left(F, G, \omega_{f}\right)$ the couple of functions $H^{\prime}(x, t)=\left(u^{\prime}(x, t), p^{\prime}(x, t)\right)$, given by

$$
\begin{aligned}
& u^{\prime}(x, t)=h(t) \\
& \cdot \int_{0}^{t}\left[\left(F\left[x-\left(U_{0}-c_{0}\right)(t-\tau)\right]\right.\right. \\
& \left.+F\left[x-\left(U_{0}+c_{0}\right)(t-\tau)\right]\right) \times(2)^{-1} \\
& +\left(G\left[x-\left(U_{0}+c_{0}\right)(t-\tau)\right]\right. \\
& \left.\left.-G\left[x-\left(U_{0}-c_{0}\right)(t-\tau)\right]\right) \times\left(2 c_{0} \rho_{0}\right)^{-1}\right] \\
& \cdot \sin \omega_{f} \tau d \tau \\
& p^{\prime}(x, t)=h(t) \\
& \cdot \int_{0}^{t}\left[c _ { 0 } \rho _ { 0 } \cdot \left(F\left[x-\left(U_{0}+c_{0}\right)(t-\tau)\right]\right.\right. \\
& \left.-F\left[x-\left(U_{0}-c_{0}\right)(t-\tau)\right]\right) \times(2)^{-1} \\
& +\left(G\left[x-\left(U_{0}+c_{0}\right)(t-\tau)\right]\right. \\
& \left.\left.+G\left[x-\left(U_{0}-c_{0}\right)(t-\tau)\right]\right) \times(2)^{-1}\right] \\
& \cdot \sin \omega_{f} \tau d \tau
\end{aligned}
$$

is continuously differentiable and verifies (5) and the condition

$$
H^{\prime}(x, t)=\left(u^{\prime}(x, t), p^{\prime}(x, t)\right)=(0,0) \quad \text { for } t \leq 0 .
$$

Proposition 5. In the class of the continuously differentiable functions, the couple of functions $\left(u^{\prime}(x, t), p^{\prime}(x, t)\right)$ given by (14), is the unique point wise solution of the problem (5), (13), and (15).

Proof. The proof of this proposition is similar to the proof of Proposition 3.

In the following the couple of functions defined by (14) is considered to be the solution of the problem of propagation of the source-produced permanent time harmonic perturbation in case of the function space $X_{1}$.

Proposition 6. For $T>0, \varepsilon>0$, and $\delta=\delta(\varepsilon, T)=\varepsilon / 2 T$ $\max \left(1+c_{0} \rho_{0}, 1+\left(1 / c_{0} \rho_{0}\right)\right)>0$ if $H=(F, G) \in V_{0}^{\delta}$, then $H^{\prime}(\circ, t) \in V_{0}^{\varepsilon}$, for any $t \in[0, T]$.

Proof. The proof of this proposition is similar to the proof of Proposition 4.

This means that in this function space the propagation problem (5) and (13), of the source-produced permanent time harmonic perturbation, is well posed on $[0, T]$, for any $T>0$.

Concerning the linear stability of the constant flow, the following statement holds. 
Proposition 7. In the case of source produced permanent time harmonic perturbations whose amplitude belongs to the function space $X_{1}$ the constant flow is linearly unstable.

Proof. The linear instability of the constant flow $U_{0}, p_{0}, \rho_{0}$ means that there exists $\varepsilon>0$ such that for any $\delta>0$ there exist $H=(F, G) \in V_{0}^{\delta}$ and $t \geq 0$ such that the corresponding solution $H^{\prime}(x, t)$ (given by (14)) satisfies $H^{\prime}(\cdot, t) \notin V_{0}^{\varepsilon}$.

The instability can be seen considering for instance $\varepsilon=1$, the perturbation $P_{\delta}\left(F, G, \omega_{f}\right)=h(t) \cdot\left(\delta \cdot \sin x \cdot \sin \omega_{f} t, 0\right)$ where $\delta>0$ is an arbitrary real number and $\omega_{f}=c_{0}-U_{0}$. Note that by choosing $\delta$ small, the above perturbation can be made as small as we wish. According to (14), the propagation of this perturbation is given by

$$
\begin{aligned}
& u^{\prime}(x, t)=h(t) \cdot \frac{\delta}{4} \\
& \left\{-t \cdot \cos \left(x+\omega_{f} t\right)+\frac{\sin 2 \omega_{f} t}{2 \omega_{f}}\right. \\
& \cdot \cos \left(x+\omega_{f} t\right)+\frac{\sin ^{2} \omega_{f} t}{\omega_{f}} \cdot \sin \left(x+\omega_{f} t\right) \\
& +\left[\frac{\sin 2\left(\omega_{f}-c_{0}\right) t}{2\left(\omega_{f}-c_{0}\right)}-\frac{\sin 2 c_{0} t}{2 c_{0}}\right] \\
& \cdot \cos \left(x+\left(\omega_{f}-2 c_{0}\right) t\right) \\
& +\left[\frac{\sin ^{2}\left(\omega_{f}-c_{0}\right) t}{2\left(\omega_{f}-c_{0}\right)}+\frac{\sin ^{2} c_{0} t}{c_{0}}\right] \\
& \left.\cdot \sin \left(x+\left(\omega_{f}-2 c_{0}\right) t\right)\right\} \text {, } \\
& p^{\prime}(x, t)=h(t) \cdot \frac{c_{0} \rho_{0} \delta}{4} \\
& \left\{t \cdot \cos \left(x+\omega_{f} t\right)-\frac{\sin 2 \omega_{f} t}{2 \omega_{f}}\right. \\
& \cdot \cos \left(x+\omega_{f} t\right)-\frac{\sin ^{2} \omega_{f} t}{\omega_{f}} \cdot \sin \left(x+\omega_{f} t\right) \\
& +\left[\frac{\sin 2\left(\omega_{f}-c_{0}\right) t}{2\left(\omega_{f}-c_{0}\right)}-\frac{\sin 2 c_{0} t}{2 c_{0}}\right] \\
& \cdot \cos \left(x+\left(\omega_{f}-2 c_{0}\right) t\right) \\
& +\left[\frac{\sin ^{2}\left(\omega_{f}-c_{0}\right) t}{2\left(\omega_{f}-c_{0}\right)}+\frac{\sin ^{2} c_{0} t}{c_{0}}\right] \\
& \left.\cdot \sin \left(x+\left(\omega_{f}-2 c_{0}\right) t\right)\right\} \text {. }
\end{aligned}
$$

Hence, for a given $\delta$, if $t$ is sufficiently large, then $H^{\prime}(\cdot, t) \notin V_{0}^{1}$ (that is because $\left(u^{\prime}(\cdot, t), p^{\prime}(\cdot, t)\right)$ is as large as we wish).

The Briggs-Bers stability analysis $[14,15]$, with respect to source-produced permanent time harmonic perturbation whose amplitude belongs to $X_{1}$, cannot be applied. That is because in this context dispersion relations cannot be derived (there exist perturbations whose amplitude has no Fourier transform; for instance $F(x)=G(x)=\exp (x))$. So, it is impossible to analyze the imaginary parts of the zeros of the dispersion relations as requires the Briggs-Bers stability criterion.

It follows that the above instability cannot be obtained by Briggs-Bers stability analysis.

\section{The Second Function Space}

The set $X_{2}$ of the perturbations of the initial value is the normed space [16] of the couples $H=(F, G)$ of continuously differentiable and bounded functions $F, G: R^{\prime} \rightarrow R^{\prime}$ with respect to the usual algebraic operations and norm defined by

$$
\|H\|=\max \left\{\sup _{x \in R^{\prime}}|F(x)|, \sup _{x \in R^{\prime}}|G(x)|\right\} .
$$

The set $V_{0}^{\varepsilon}$, defined by:

$$
V_{0}^{\varepsilon}=\left\{H=(F, G) \in X_{2}:\|H\|<\varepsilon\right\},
$$

is a neighborhood of the origin and the meaning of the concept "the perturbation $H=(F, G) \in X_{1}$ is small" is that there exists $\varepsilon>0$ small such that $H \in V_{0}^{\varepsilon}$.

For an initial data $H=(F, G) \in X_{2}$ the couple of functions $H^{\prime}(x, t)=\left(u^{\prime}(x, t), p^{\prime}(x, t)\right)$, given by $(7)$, is the unique bounded classical solution of (4) and (8).

If a sequence of initial data $H_{n}=\left(F_{n}, G_{n}\right) \in X_{2}$ converges in $X_{2}$ to $H=(F, G) \in X_{2}$, then for any $t \geq 0$ (fixed) the sequence of the corresponding solutions $H_{n}^{\prime}(\cdot, t)$ converges in $X_{2}$ to the solution $H^{\prime}(\cdot, t)$ corresponding to $H$.

This means that for the set of initial data $X_{2}$, the initial value problem (4) and (19)

$$
\left(u^{\prime}(x, 0), p^{\prime}(x, 0)\right)=H^{\prime}(x, 0)=H(x)=(F(x), G(x))
$$

is well posed in sense of Hadamard $[5,6]$ on $[0, T]$, for any $T>0$.

In the following the couple of functions defined by (7) is considered to be the solution to the problem of propagation of the initial value perturbation for data in $X_{2}$.

The linear stability of the constant flow $U_{0}, p_{0}, \rho_{0}$ would mean that for any $\varepsilon>0$ there exists $\delta=\delta(\varepsilon)$ such that for any $H \in X_{2}$ if $\|H\|<\delta(\varepsilon)$ the corresponding solution $H^{\prime}(x, t)$ (given by (7)) satisfies $\left\|H^{\prime}(\cdot, t)\right\|<\varepsilon$ for any $t \geq 0$.

Concerning the linear stability, the following statement holds.

Proposition 8. For any $\varepsilon>0$ and $H \in X_{2}$ if $\|H\|<\varepsilon / 2$ max $\left(1+c_{0} \rho_{0}, 1+1 / c_{0} \rho_{0}\right)$, then $\left\|H^{\prime}(\cdot, t)\right\|<\varepsilon$ for $t \geq 0$. 
Proof. The proof is similar to that of Proposition 4.

This means that the null solution of (4) is stable, that is, the constant solution $u=U_{0}, p=p_{0}, \rho=\rho_{0}$ of (1), is linearly stable, with respect to the initial value perturbation with data from $X_{2}$.

For initial data from $X_{2}$ the exponential growth rate, given by (12), of the solution of the initial value problem (4) and (19) is equal to zero. However, for initial values from $X_{2}$ the Briggs-Bers stability analysis, in case of the problem (4) and (19), cannot be applied. That is because, for instance, the Fourier transform with respect to $x$ of the initial value $F(x)=$ $G(x) \equiv 1$ does not exist. So, dispersion relations cannot be derived and the stability criterion cannot be used. Therefore, the above stability results cannot be obtained by Briggs-Bers stability analysis.

For source-produced permanent time harmonic perturbations, whose amplitudes belong to $X_{2}$, the right-hand members $Q_{1}, Q_{2}$, appearing in (5), are those given by (13) with $(F, G) \in X_{2}$.

For the perturbation $P\left(F, G, \omega_{f}\right)\left((F, G) \in X_{2}\right)$ the couple of functions $H^{\prime}(x, t)=\left(u^{\prime}(x, t), p^{\prime}(x, t)\right)$, given by $(14)$, is the unique solution of the problem (5), (13), and (15) satisfying

$$
H^{\prime}(x, t)=\left(u^{\prime}(x, t), p^{\prime}(x, t)\right)=(0,0) \quad \text { for } t \leq 0 .
$$

Concerning the continuous dependence on the amplitude of the perturbation, the following statement holds.

Proposition 9. For any $T>0, \varepsilon>0$ and $\delta=\delta(\varepsilon, T)=\varepsilon / 2 T$ $\max \left(1+c_{0} \rho_{0}, 1+\left(1 / c_{0} \rho_{0}\right)\right)>0$ if $\|H\|_{X_{2}}<\delta$, then $\left\|H^{\prime}(\cdot, t)\right\|_{X_{2}}$ $<\varepsilon$ for any $t \in[0, T]$.

Proof. The proof is similar to the proof of Proposition 6.

This means that the problem (5) and (20) is well posed on $[0, T]$, for any $T>0$.

When the amplitude of the source-produced perturbation belongs to the function space $X_{2}$ the couple of functions defined by (14) is considered to be the solution of the problem of propagation of the source-produced permanent time harmonic perturbation.

Concerning the linear stability of the constant flow, the following statement holds.

Proposition 10. The constant flow of (1) is linearly unstable with respect to the source-produced permanent time harmonic perturbations whose amplitude belongs to the function space $X_{2}$.

Proof. It turns that the perturbations $P_{\delta}\left(F, G, \omega_{f}\right)=h(t)$. $\left(\delta \cdot \sin x \cdot \sin \omega_{f} t, 0\right)$ considered in the function space $X_{1}$ are appropriate to show the above statement.

The Briggs-Bers stability analysis [14, 15], with respect to source-produced permanent time harmonic perturbation whose amplitude belongs to $X_{2}$, can not be applied. That is because in this context dispersion relations can not be derived (there exist perturbations whose amplitude has no Fourier transform; for instance, the perturbation $h(t) \cdot(\sin x$. $\left.\sin \omega_{f} t, 0\right)$ has no Fourier transform with respect to $x$ ). So, it is impossible to analyze the imaginary parts of the zeros of the dispersion relations as requires the Briggs-Bers stability criterion. Therefore, the above instability result cannot be obtained by Briggs-Bers stability analysis.

\section{The Third Function Space}

In this case the set $X_{3}$ of the initial data is the normed space of the couples $H=(F, G)$ of functions $F, G \in L^{2}\left(R^{\prime}\right)$ [17] with respect to the usual algebraic operations and the norm defined by

$$
\|H\|_{X_{3}}=\max \left\{\left(\int_{R^{\prime}}|F(x)|^{2} d x\right)^{1 / 2},\left(\int_{R^{\prime}}|G(x)|^{2} d x\right)^{1 / 2}\right\} .
$$

It has to be emphasized that the functions $F, G \in L^{2}\left(R^{\prime}\right)$ are defined up to addition of a measure zero function.

The meaning of the concept "the perturbation $H=(F, G)$ $\epsilon X_{3}$ is small" is that there exists $\varepsilon>0$ small such that $\|H\|_{X_{3}}<\varepsilon$.

For an initial data $H=(F, G) \in X_{3}$ the couple of functions $H^{\prime}(x, t)=\left(u^{\prime}(x, t), p^{\prime}(x, t)\right)$, given by (7), is called generalized solution of (4). A generalized solution is unique up to addition of a measure zero solution. In general, $H^{\prime}(x, t)$ is not differentiable but $H^{\prime}(\cdot, t) \in X_{3}$ and $H^{\prime}(x, 0)$ satisfy the condition

$$
H^{\prime}(x, 0)=\left(u^{\prime}(x, 0), p^{\prime}(x, 0)\right)=(F, G) .
$$

When $F, G$ are continuously differentiable, then $H^{\prime}(x, t)$ satisfies (4) in classical sense.

It has to be noted that there exist continuous functions $F, G$ which are not differentiable at every point [17]. The generalized solution which corresponds to an initial data of this type is continuous but it is not differentiable at every point.

Concerning the continuous dependence on the initial data, we remark that for $t$ fixed $H^{\prime}(\cdot, t)$ satisfies

$$
\left\|H^{\prime}(\cdot, t)\right\|_{X_{3}} \leq \max \left\{1+c_{0} \rho_{0}, 1+\frac{1}{c_{0} \rho_{0}}\right\} \cdot\left\|H^{\prime}(\cdot, 0)\right\|_{X_{3}} .
$$

Inequality (23) implies that if the sequence of initial data $H_{n}=$ $\left(F_{n}, G_{n}\right) \in X_{3}$ converges in $X_{3}$ to $H=(F, G) \in X_{3}$, then for any $t \geq 0$ the sequence of the corresponding solutions $H_{n}^{\prime}(\cdot, t)$ converges in $X_{3}$ to $H^{\prime}(\cdot, t)$, corresponding to $H=(F, G) \in$ $X_{3}$.

This means continuous dependence on the initial data.

Therefore, in the case of the set of initial data $X_{3}$, the initial value problem (4) and (22) is well posed in sense of Hadamard on $[0, T]$ for every $T>0$.

In the following the couple of functions defined by (7) up to addition of a measure zero solution, is considered to be the solution to the problem of propagation of the initial value perturbation for data in $X_{3}$.

As concerns stability, the following statement holds. 
Proposition 11. For any $\varepsilon>0$ and $H \in X_{3}$ if $\|H\|<\varepsilon / 2$ max $\left(1+c_{0} \rho_{0}, 1+1 / c_{0} \rho_{0}\right)$, then $\left\|H^{\prime}(\cdot, t)\right\|<\varepsilon$ for $t \geq 0$.

Proof. The proof is similar to that of Proposition 8.

This means that the null solution of (4) is stable; that is, the constant solution $=U_{0}, p=p_{0}, \rho=\rho_{0}$ of (1) is linearly stable with respect to the initial value perturbation with data from $X_{3}$.

For initial data from $X_{3}$ the exponential growth rate, given by (12), of the solution of the initial value problem (4) and (22), is equal to zero.

For every generalized solution the Laplace transform is defined for $\operatorname{Re} z>0$ and the Fourier transform with respect to $x$ exists. So, dispersion relation can be derived and the stability can be analyzed by analyzing the imaginary parts of the zeros of the dispersion relation. This means that both steps of the Briggs-Bers stability analysis can be undertaken. However, the above stability result was obtained directly not by Briggs-Bers stability analysis.

What can be strange for engineers in this function space is the presence of solutions which are continuous but are not differentiable at every point. Which kind of propagation does represent such a solution?

For source-produced permanent time harmonic perturbation, whose amplitudes belong to $X_{3}$, the right-hand members $Q_{1}, Q_{2}$, appearing in (5), are those given by (13) with $(F, G) \in X_{3}$.

For the perturbation $P\left(F, G, \omega_{f}\right)\left((F, G) \in X_{3}\right)$ the couple of functions $H^{\prime}(x, t)=\left(u^{\prime}(x, t), p^{\prime}(x, t)\right)$, given by (14) up to addition of a measure zero solution will be called generalized solution of the propagation problem (5) and (24)

$$
H^{\prime}(x, t)=\left(u^{\prime}(x, t), p^{\prime}(x, t)\right)=(0,0) \quad \text { for } t \leq 0 \text {. }
$$

Concerning the continuous dependence on the amplitude of the perturbation, the following statement holds.

Proposition 12. For any $T>0, \varepsilon>0$ and $\delta=\delta(\varepsilon, T)=\varepsilon / 2 T$ $\max \left(1+c_{0} \rho_{0}, 1+\left(1 / c_{0} \rho_{0}\right)\right)>0$ if $\|H\|_{X_{3}}<\delta$, then $\left\|H^{\prime}(\cdot, t)\right\|_{X_{3}}<$ $\varepsilon$ for any $t \in[0, T]$.

Proof. The proof is similar to the proof of Proposition 6.

This means that the problem (5) and (24) is well posed on $[0, T]$ for any $T>0$.

The linear instability of the constant flow of (1) with respect to this kind of perturbations can be shown similarly as in the function space $X_{1}$, by considering the perturbations $F(x)=\delta \sin x / 1+x^{2}, G(x)=0$ and $\omega_{f}=c_{0}-U_{0}$.

Since the Fourier transform with respect to $x$ and the Laplace transform with respect to $t$ of any solution exist, dispersion relation can be derived, and the stability analysis could be undertaken by analyzing the imaginary parts of zeros of dispersion relations (both steps of the Briggs-Bers stability analysis can be undertaken in this function space). However, in this paper the instability was derived directly.

\section{The Fourth Function Space}

The set $X_{4}$ of the perturbations of the initial data is the locally convex vector space of the couples $H=(F, G)$ of tempered distributions $F, G \in S^{\prime}\left(R^{\prime}\right)$ [8] with respect to the usual algebraic operations and seminorms $\widetilde{q}_{B}$ defined by

$$
\tilde{q}_{B}(H)=\max \left\{q_{B}(F), q_{B}(G)\right\},
$$

where $B$ are bounded sets in the space of the rapidly decreasing functions $S\left(R^{\prime}\right)$ and $q_{B}$ is the seminorm on $S^{\prime}\left(R^{\prime}\right)$ defined by $q_{B}(F)=\sup _{\varphi \in B}|F(\varphi)|$.

The set $V_{0}^{\varepsilon, \widetilde{q}_{B}}$ defined by

$$
V_{0}^{\varepsilon, \widetilde{q}_{B}}=\left\{H=(F, G) \in X_{4}: \widetilde{q}_{B}(H)<\varepsilon\right\}
$$

is a neighborhood of the origin and the meaning of the concept "the perturbation $H=(F, G) \in X_{4}$ is small" is that there exists $\varepsilon>0$ small and $B$ bounded such that $H=(F, G) \in$ $V_{0}^{\varepsilon, \tilde{q}_{B}}$.

If the initial value perturbation is a couple of tempered distributions $H=(F, G) \in X_{4}$, then a solution of (4) is a family $H^{\prime}(t)=\left(u^{\prime}(t), p^{\prime}(t)\right)$ of couples of tempered distributions which satisfies (4) for $t \geq 0$ and the initial condition

$$
H^{\prime}(0)=\left(u^{\prime}(0), p^{\prime}(0)\right)=H=(F, G) \in X_{4} .
$$

Proposition 13. If the initial value problem (4) and (27) has a solution $H^{\prime}(t)=\left(u^{\prime}(t), p^{\prime}(t)\right)$, then its Fourier transform with respect to $x$ is a family of couples of tempered distributions $\widehat{H}^{\prime}(t)=\left(\widehat{u}^{\prime}(t), \widehat{p}^{\prime}(t)\right), t \geq 0$ which satisfies the following equations:

$$
\begin{aligned}
& \frac{\partial \widehat{u}^{\prime}}{\partial t}+i k U_{0} \cdot \widehat{u}^{\prime}+i k \frac{1}{\rho_{0}} \cdot \widehat{p}^{\prime}=0, \\
& \frac{\partial \widehat{p}^{\prime}}{\partial t}+\gamma p_{0} i k \cdot \widehat{u}^{\prime}+i k U_{0} \cdot \widehat{p}^{\prime}=0
\end{aligned}
$$

and the initial condition

$$
\widehat{u}^{\prime}(0)=\widehat{F}, \quad \widehat{p}^{\prime}(0)=\widehat{G} .
$$

Proof. By computation.

Remark, that the problem (4) and (27) is well posed if and only if the problem (28) and (29) is well posed.

Concerning the problem (28) and (29) the following statement holds.

Proposition 14. The problem (28) and (29) is well posed on any finite interval and its solutions is given by

$$
\begin{aligned}
\hat{u}^{\prime}(t)= & \frac{e^{-i\left(U_{0}-c_{0}\right) k t}+e^{-i\left(U_{0}+c_{0}\right) k t}}{2} \\
& \cdot \widehat{F}+\frac{e^{-i\left(U_{0}+c_{0}\right) k t}-e^{-i\left(U_{0}-c_{0}\right) k t}}{2 c_{0} \rho_{0}} \cdot \widehat{G}, \\
\widehat{p}^{\prime}(t)= & c_{0} \rho_{0} \frac{e^{-i\left(U_{0}+c_{0}\right) k t}-e^{-i\left(U_{0}-c_{0}\right) k t}}{2} \\
& \cdot \widehat{F}+\frac{e^{-i\left(U_{0}+c_{0}\right) k t}+e^{-i\left(U_{0}-c_{0}\right) k t}}{2} \cdot \widehat{G} .
\end{aligned}
$$


Proof. The existence and uniqueness are easy to see. We will show only the continuous dependence on the initial data in case of the problem (28) and (29). For that, consider $T>0, \varepsilon>0$ and a semi norm $\widetilde{q}_{B}$ in $X_{4}$. After that, consider a bounded set $B^{\prime} \subset S\left(R^{\prime}\right)$, which contains the following bounded sets:

$$
\begin{gathered}
\frac{e^{-i\left(U_{0}-c_{0}\right) k t}+e^{-i\left(U_{0}+c_{0}\right) k t}}{2} \cdot B ; \\
\frac{e^{-i\left(U_{0}+c_{0}\right) k t}-e^{-i\left(U_{0}-c_{0}\right) k t}}{2 c_{0} \rho_{0}} \cdot B ; \\
c_{0} \rho_{0} \frac{e^{-i\left(U_{0}+c_{0}\right) k t}-e^{-i\left(U_{0}-c_{0}\right) k t}}{2} \cdot B
\end{gathered}
$$

for any $t \in[0, T]$; the semi norm $\widetilde{q}_{B^{\prime}}$ in $X_{4}$ and $\delta=\varepsilon /(1+$ $\left.\widetilde{q}_{B^{\prime}}(\widehat{F}, \widehat{G})\right)>0$. Remark that for any $(\widehat{F}, \widehat{G}) \in X_{4}$ with $\widetilde{q}_{B^{\prime}}$ $(\widehat{F}, \widehat{G})<\delta$ we have $\widetilde{q}_{B}\left(\widehat{u}^{\prime}(t), \widehat{p}^{\prime}(t)\right)<\varepsilon$ for $t \in[0, T]$.

Proposition 15. The problem (4) and (27) is well posed.

Proof. The inverse Fourier transform of $\left(\widehat{u}^{\prime}(t), \widehat{p}^{\prime}(t)\right)=\widehat{H}^{\prime}(t)$ given by (30) is the unique solution of (4) and (27). The continuous dependence of the solutions of (4) and (27) is a corollary of the continuous dependence on the initial data of the solution of (28) and (29) and of the continuity of the Fourier and inverse Fourier transforms.

So, the problem (4) and (27) is well posed.

Concerning the stability, the following statement holds.

Proposition 16. The null solution of (28) is unstable.

Proof. The instability of the null solution of (28) means that there exist $\varepsilon_{0}>0$ and a semi norm $\widetilde{q}_{B_{0}}$ such that for any real number $\delta>0$ and any semi norm $\widetilde{q}_{B}$ in $X_{4}$ there exist $H=$ $(F, G) \in V_{0}^{\delta, \widetilde{q}_{B}}$ and $t \geq 0$ such that the corresponding solution $H^{\prime}(t)$ (given by (30)) satisfies $H^{\prime}(t) \notin V_{0}^{\varepsilon_{0}, \widetilde{q}_{B_{0}}}$. Let us consider now $\varepsilon_{0}=1, B_{0}$-a bounded set of rapidly decreasing functions which contains the function $\varphi_{0}(k)=\exp \left(-k^{2}\right)$, and the tempered distribution defined by $\widehat{F}_{0}(\varphi)=\varphi^{\prime}(0)$. Remark that for an arbitrary real number $\delta>0$ and an arbitrary bounded set $B$ of rapidly decreasing functions, there exists a real number $\varepsilon>0$ such that $q_{B}\left(\varepsilon \widehat{F}_{0}\right)<\delta\left(V_{0}^{\delta, q_{B}}\right.$ is absorbing). It follows that $\left(\varepsilon \cdot \widehat{F}_{0}, 0\right) \in V_{0}^{\delta, \widetilde{q}_{B}}$. On the other hand, for the solution $\left(\widehat{u}^{\prime}(t), \widehat{p}^{\prime}(t)\right)$ of the initial value problem (28), (29) with $\widehat{F}=\varepsilon \widehat{F}_{0}$ and $\widehat{G}=0$, the following relations hold:

$$
\begin{gathered}
\widehat{u}^{\prime}(t)=-i \varepsilon U_{0} t \varphi(0)+\varphi^{\prime}(0), \\
\widehat{p}^{\prime}(t)=-i \varepsilon c_{0}^{2} \rho_{0} t \varphi(0), \\
q_{B_{0}}\left(\widehat{u}^{\prime}(t)\right)=\varepsilon \cdot \sup _{\varphi \in B_{0}}\left|\widehat{u}^{\prime}(t)(\varphi)\right| \geq \varepsilon \\
\cdot\left|\widehat{u}^{\prime}(t)\left(\varphi_{0}\right)\right|=\varepsilon U_{0} t,
\end{gathered}
$$

$$
\begin{array}{r}
q_{B_{0}}\left(\widehat{p}^{\prime}(t)\right)=\varepsilon \cdot \sup _{\varphi \in B_{0}}\left|\widehat{p}^{\prime}(t)(\varphi)\right| \geq \varepsilon \\
\cdot\left|\widehat{p}^{\prime}(t)\left(\varphi_{0}\right)\right|=\varepsilon c_{0}^{2} \rho_{0} t, \\
\widetilde{q}_{B_{0}}\left(\widehat{u}^{\prime}(t), \widehat{p}^{\prime}(t)\right) \geq \varepsilon t \max \left\{U_{0}, c_{0}^{2} \rho_{0}\right\} .
\end{array}
$$

Therefore, for $t$ sufficiently large, we have $\widetilde{q}_{B_{0}}\left(\widehat{u}^{\prime}(t), \widehat{p}^{\prime}(t)\right)>$ $1=\varepsilon_{0}$.

Hence, the null solution of (28) is unstable. Since the inverse Fourier transform is continuous, the null solution of (4) is also unstable in $X_{4}$.

For source-produced permanent time harmonic perturbations, whose amplitudes belong to $X_{4}$, the right-hand members $Q_{1}, Q_{2}$, appearing in (5) are given by

$$
Q_{1}=h(t) \cdot e^{i \omega_{f} t} \cdot F, \quad Q_{2}=h(t) \cdot e^{i \omega_{f} t} \cdot G
$$

where $H=(F, G) \in X_{4}$.

The unknown tempered distributions $u^{\prime}(t), p^{\prime}(t)$ have to satisfy (5) and the condition

$$
u^{\prime}(t)=0, \quad p^{\prime}(t)=0, \quad \text { for } t \leq 0
$$

If the problem (5) and (34) has a solution $H^{\prime}(t)=\left(u^{\prime}(t)\right.$, $\left.p^{\prime}(t)\right) \in X_{4}$, then the Fourier transforms $\widehat{u}^{\prime}(t), \widehat{v}^{\prime}(t)$ are tempered distributions and satisfy

$$
\begin{gathered}
\frac{\partial \widehat{u}^{\prime}}{\partial t}+i k U_{0} \cdot \widehat{u}^{\prime}+i k \frac{1}{\rho_{0}} \cdot \widehat{p}^{\prime}=h(t) \cdot e^{i \omega_{f} t} \cdot \widehat{F} \\
\frac{\partial \widehat{p}^{\prime}}{\partial t}+\gamma p_{0} i k \cdot \widehat{u}^{\prime}+i k U_{0} \cdot \widehat{p}^{\prime}=h(t) \cdot e^{i \omega_{f} t} \cdot \widehat{G} \\
\widehat{u}^{\prime}(t)=0, \quad \widehat{p}^{\prime}(t)=0 \quad \text { for } t \leq 0 .
\end{gathered}
$$

Hence, we got

$$
\begin{aligned}
\widehat{u}^{\prime}(t)= & \frac{h(t)}{2} \\
& \times\left[\frac{e^{i \omega_{f} t}-e^{-i\left(U_{0}-c_{0}\right) k t}}{i\left[\left(U_{0}-c_{0}\right) \cdot k+\omega_{f}\right]}\right. \\
& \left.+\frac{e^{i \omega_{f} t}-e^{-i\left(U_{0}+c_{0}\right) k t}}{i\left[\left(U_{0}+c_{0}\right) \cdot k+\omega_{f}\right]}\right] \cdot \widehat{F} \\
+ & \frac{h(t)}{2 c_{0} \rho_{0}}\left[\frac{e^{i \omega_{f} t}-e^{-i\left(U_{0}+c_{0}\right) k t}}{i\left[\left(U_{0}+c_{0}\right) \cdot k+\omega_{f}\right]}\right. \\
& \left.\quad-\frac{e^{i \omega_{f} t}-e^{-i\left(U_{0}-c_{0}\right) k t}}{i\left[\left(U_{0}-c_{0}\right) \cdot k+\omega_{f}\right]}\right] \cdot \widehat{G},
\end{aligned}
$$




$$
\begin{aligned}
& \hat{p}^{\prime}(t)= \frac{c_{0} \rho_{0} \cdot h(t)}{2} \\
& \times {\left[\frac{e^{i \omega_{f} t}-e^{-i\left(U_{0}+c_{0}\right) k t}}{i\left[\left(U_{0}+c_{0}\right) \cdot k+\omega_{f}\right]}\right.} \\
&\left.-\frac{e^{i \omega_{f} t}-e^{-i\left(U_{0}-c_{0}\right) k t}}{i\left[\left(U_{0}-c_{0}\right) \cdot k+\omega_{f}\right]}\right] \cdot \widehat{F} \\
&+\frac{h(t)}{2}\left[\frac{e^{i \omega_{f} t}-e^{-i\left(U_{0}+c_{0}\right) k t}}{i\left[\left(U_{0}+c_{0}\right) \cdot k+\omega_{f}\right]}\right. \\
&\left.\quad+\frac{e^{i \omega_{f} t}-e^{-i\left(U_{0}-c_{0}\right) k t}}{i\left[\left(U_{0}-c_{0}\right) \cdot k+\omega_{f}\right]}\right] \cdot \widehat{G} .
\end{aligned}
$$

It can be seen that for arbitrary $F, G \in S^{\prime}\left(R^{\prime}\right), \widehat{u}^{\prime}(t), \widehat{v}^{\prime}(t)$, given by (36), are not necessarily tempered distributions. This contradiction shows that the problem (5) and (34) is ill posed.

\section{Conclusions}

(i) The obtained results show that the well-posedness and linear stability concepts of the constant gas flow in 1D flow model are highly dependent on the function space. There exists function space in which the constant gas flow is stable and another function space in which the same flow is unstable with respect to the initial value perturbations. There exists function space in which the propagation problem of the source produced perturbation is well posed and another function space in which the propagation problem is ill posed.

(ii) For the stability of the constant gas flow with respect to the initial value perturbations it is not necessary that the set of the exponential growth rate of the solutions of the homogeneous linearized Euler equations be bounded from above. There exists function space in which the constant gas flow is stable with respect to the initial value perturbations although the set of the exponential growth rate of the solutions of the homogeneous Euler equations is the whole real axis. So, the stability of the null solution cannot be denied just because the set of the exponential growth rate of the solutions is not bounded from above.

(iii) The stability of the constant gas flow with respect to the initial value perturbations is not a sufficient condition for the stability of the same solution with respect to source produced permanent time harmonic perturbations. There exists function space in which the constant gas flow is stable with respect to the initial value perturbations and it is unstable with respect to the source produced perturbations.

(iv) In two of the considered function spaces the BriggsBers stability analysis cannot be applied, because dispersion relations cannot be derived. So, the stability or instability results obtained directly in this function spaces cannot be obtained by Briggs-Bers stability analysis.

(v) Continuous dependence on the initial data (on the source amplitude, resp.) is an expression of stability on a finite interval of time and stability means continuous dependence on the infinite interval of time $[0,+\infty)$. In practice, the duration of the propagation is finite. For the eight situations considered in this paper, the continuous dependence on any finite interval of time in seven situations is valid. The stability on $[0,+\infty)$ is valid only in three situations. So, the continuous dependence on any finite interval of time is less dependent on the function space. Taking into account the above facts, from practical point of view, the question is: the continuous dependence or the stability is important?

(vi) When theoretical results are tested against experimental results, the computed mathematical variable has to correspond to the experimentally measured quantity. For instance, in this paper there are four different mathematical variables for expressing that the perturbation and the corresponding solution of the propagation problem are small, that is, close to zero. Which one of them does correspond to an experimentally measured quantity when the duct is infinitely long?

(vii) The results obtained could be useful in a better understanding of some apparently strange results published in the literature concerning the sound propagation in a gas flowing through a lined duct, since there are neither set rules nor understanding of the "right" way to model the phenomenon.

\section{Conflict of Interests}

The authors declare that there is no conflict of interests regarding the publication of this paper.

\section{Acknowledgment}

This work was supported by a grant of the Romanian National Authority for Scientific Research, CNCS-UEFISCDI, project no. PN-II-ID-PCE-2011-3-0171. The results have been presented orally at Sz.-Nagy Centennial Conference, June 2428, 2013, Szeged, Hungary (http://www.math.u-szeged.hu/ SzNagy100/program.php).

\section{References}

[1] E. J. Brambley, "Fundamental problems with the model of uniform flow over acoustic linings," Journal of Sound and Vibration, vol. 322, no. 4-5, pp. 1026-1037, 2009.

[2] E. J. Brambley, "A well-posed modified myers boundary condition," in Proceedings of the 16th AIAA/CEAS Aeroacoustics Conference (31st AIAA Aeroacoustics Conference), June 2010.

[3] Y. Renou and Y. Aurégan, "On a modified Myers boundary condition to match lined wall impedance deduced from several experimental methods in presence of a grazing flow," in Proceedings of the 16th AIAA/CEAS Aeroacoustics Conference, p. 14, June 2010.

[4] E. J. Brambley, "Well-posed boundary condition for acoustic liners in straight ducts with flow," AIAA Journal, vol. 49, no. 6, pp. 1272-1282, 2011.

[5] G. E. Ladas and V. Lakshmikantham, Differential Equations in Abstract Spaces, Academic Press, New York, NY, USA, 1972.

[6] S. G. Krein, Differential Equations in Banach Spaces, Izd. "Nauka", Moskow, 1967 (Russian). 
[7] A. M. Balint, St. Balint, and R. Szabo, "Linear stability of a non slipping gas flow in a rectangular lined duct with respect to perturbations of the initial value by indefinitely differentiable disturbances having compact support," AIP Conference Proceedings, vol. 1493, p. 1023, 2012.

[8] K. Yosida, Functional Analysis, vol. 123, Springer, Berlin, Germany, 6th edition, 1980.

[9] B. L. Rozdjestvenskii and N. N. Ianenko, Quasilinear Systems of Equations and Their Applications in Gasdynamics, Izd. "Nauka", Moskow, Russia, 1978 (Russian).

[10] P. G. Drazin and W. N. Reid, Hydrodynamic Stability, Cambridge University Press, Cambridge, UK, 1995.

[11] S. H. Saperstone, Semidynamical Systems in InfiniteDimensional Spaces, vol. 37, Springer, New York, NY, USA, 1981.

[12] N. Bourbaki, Elements de Mathematique, Premier Partie, Livre III, Topologie Generale, chapitre 1, structure topologique, Hermann, Paris, France, 1966.

[13] E. Hille and R. S. Phillips, Functional Analysis and Semi-Groups, American Mathematical Society, Providence, RI, USA, 1957.

[14] A. Bers, "Space-time evolution of plasma-instabilitiesabsolute and convective," in Handbook of Plasma Physics I, A. A. Galeev and R. M. Sudan, Eds., pp. 452-484, North Holland, Amsterdam, The Netherlands, 1983.

[15] R. J. Briggs, Electron Stream Interaction with Plasmas, Research Monograph no. 29, M.I.T. Press, Cambridge, Mass, USA, 1964.

[16] N. Dunford and J. T. Schwartz, Linear Operators, Part 1: General Theory, Wiley-Interscience, New York, NY, USA, 1957.

[17] R. Riesz and B. Sz-Nagy, Leçons d'Analyse Fonctionnelle, Akadémiai Kiadó, Budapest, Hungary, 1953. 


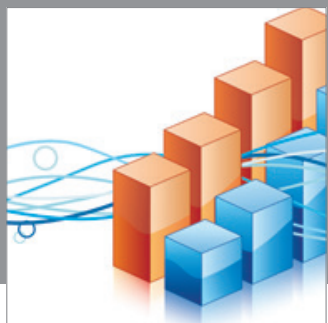

Advances in

Operations Research

mansans

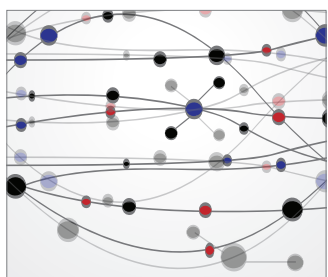

The Scientific World Journal
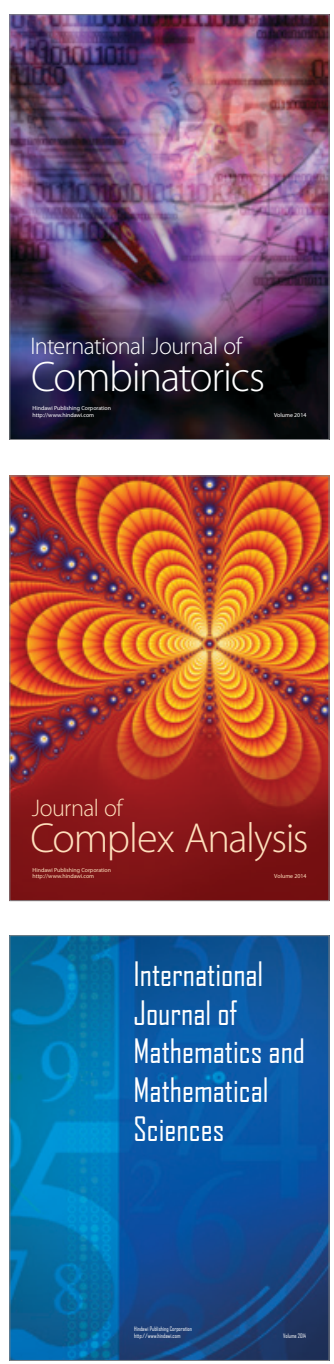
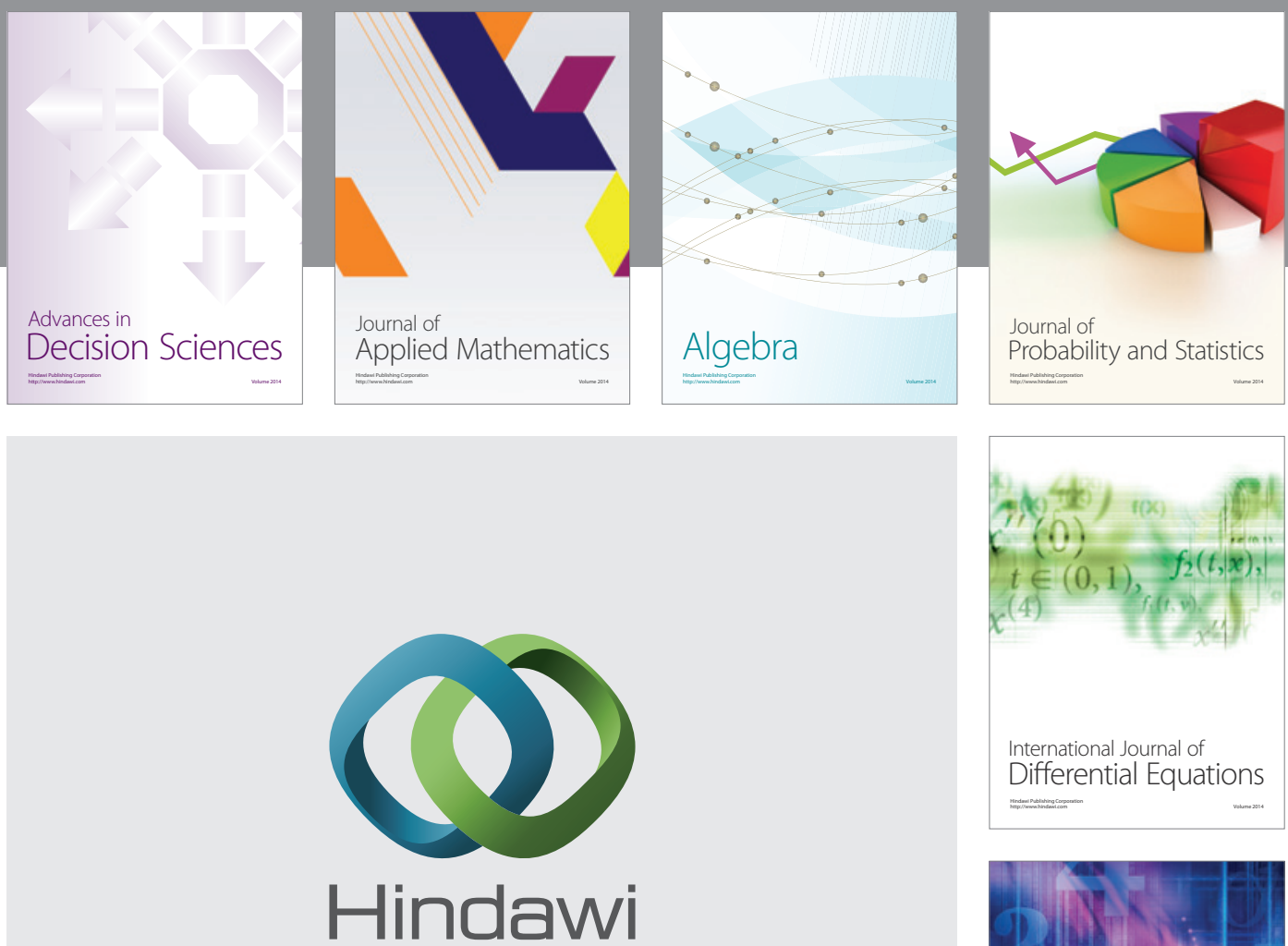

Submit your manuscripts at http://www.hindawi.com
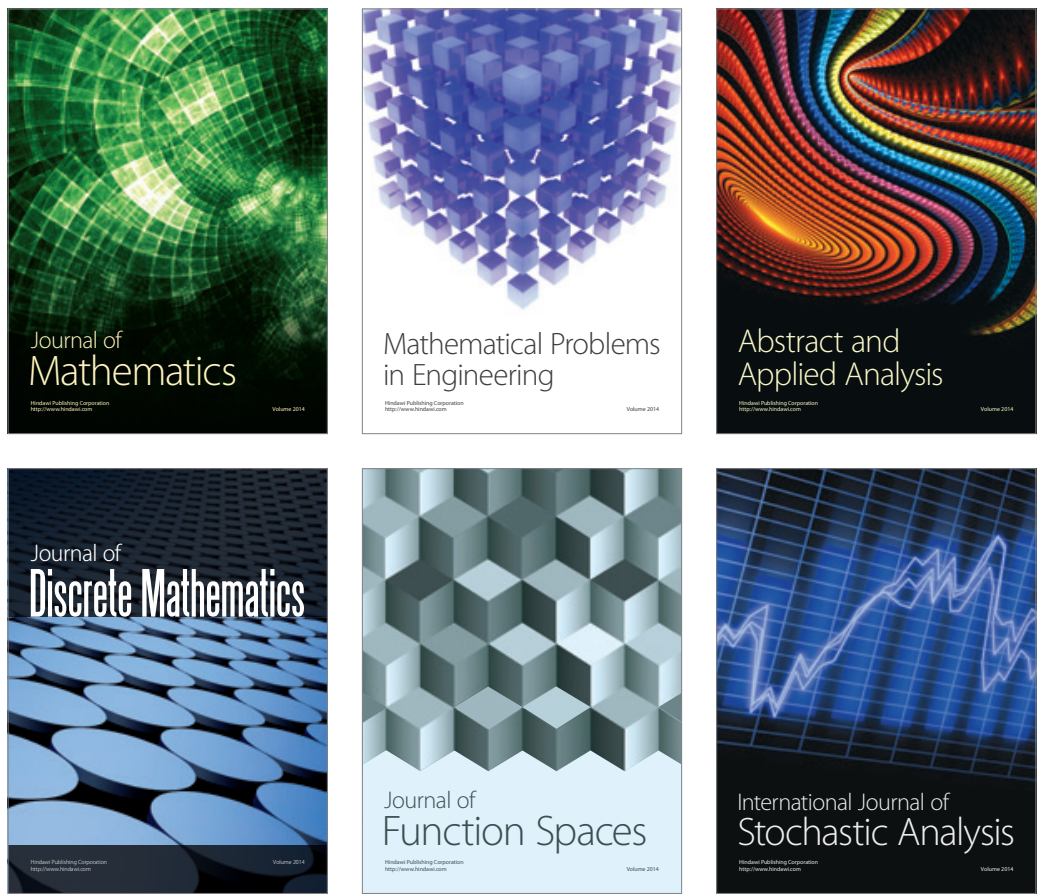

Journal of

Function Spaces

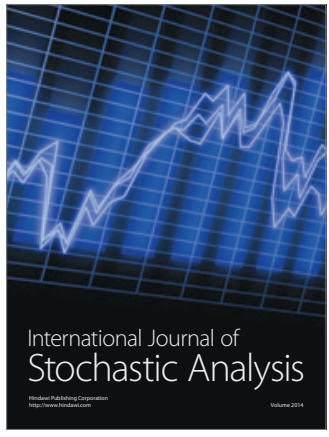

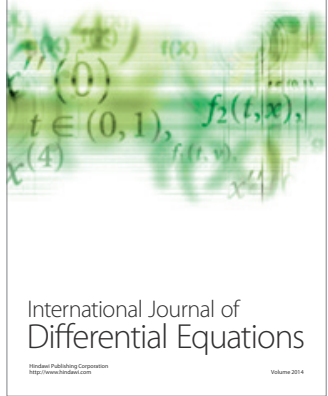
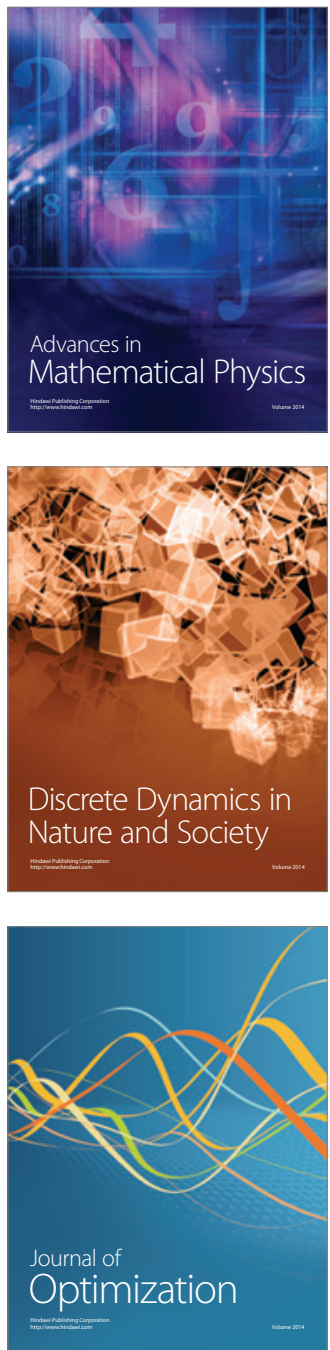